Artículos / Articles

\title{
La calidad intrínseca del trabajo y su vinculación con las lógicas laborales
}

\author{
The Intrinsic Quality of Work and its link with labor logics
}

\author{
María Cascales Mira* \\ Universidad de Sevilla, España. \\ mcascales@us.es \\ Ildefonso Marqués Perales (iD \\ Universidad de Sevilla, España. \\ imarques@us.es \\ Recibido / Received: 27/04/2020 \\ Aceptado / Accepted: 27/06/2020
}

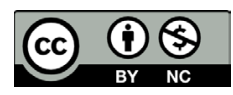

\section{RESUMEN}

Los estudios sobre calidad del trabajo han minimizado la importancia de los componentes no pecuniarios de la actividad laboral y han centrado su atención en el análisis de la estratificación social, mostrando una jerarquía vertical de la calidad laboral por clases sociales, obviando la importancia de las relaciones horizontales que subyacen a las lógicas de empleo industrial y post-industrial. El objetivo de este artículo es doble: presentar un indice compuesto que mida la calidad intrínseca del trabajo y vincularlo con las lógicas laborales. Primero se expone en qué consisten las lógicas laborales, y justificamos la necesidad de contar con un índice de Calidad Intrínseca del Trabajo. Segundo, presentamos la metodología, la construcción empírica del índice y la validación mediante análisis de clases latentes del modelo de lógicas laborales que vamos a emplear. Por último, presentamos la vinculación entre las lógicas de empleo resultantes y la calidad intrínseca del trabajo.

Palabras clave: Índice de calidad intrínseca del trabajo; lógicas laborales; calidad del trabajo; análisis de clases latentes; estratificación social.

\begin{abstract}
Studies on quality of work have minimized the importance of the non-pecuniary components of labor activity and have focused their attention on the analysis of social stratification, showing a vertical hierarchy of labor quality by social classes, obviating the importance of horizontal relationships underlying the logic of industrial and post-industrial employment. The goal of this article is twofold: to present a composite index that measures the intrinsic quality of work and link it with labor logic. First, it is explained what labor logics consist of, and we justify the need for an Intrinsic Quality of Work Index. Second, we present the methodology, the empirical construction of the index and the validation through latent class analysis of the labor logic model that we are going to use. Finally, we present the link between the resulting employment logic and the intrinsic quality of work.
\end{abstract}

Keywords: Intrinsic quality of work index; labor logics; work quality; latent class analysis; social stratification.

\footnotetext{
* Autor para correspondencia/ Corresponding author: María Cascales Mira, mcascales@us.es

Sugerencia de cita / Suggested citation: Cascales, M. y Marqués, I. (2021). La Calidad Intrínseca del Trabajo y su vinculación con las lógicas laborales. Revista Española de Sociología, 30 (1), a10. https:// doi.org/10.22325/fes/res.2021.10
} 


\section{INTRODUCCIÓN}

En este trabajo se pretende medir la calidad laboral no pecuniaria de los trabajadores según las lógicas de empleo industrial y post-industrial en las que se desenvuelven. Existe una extensa literatura que aborda la construcción de modelos de calidad laboral que se basan en indicadores que apuntan a distintas recompensas de tipo material (FernándezMacías, 2012; Hurley, Storrie y Jungblut, 2011). En cambio, los modelos de calidad laboral que miden aspectos propios del desempeño del trabajo, tan relevantes para la calidad de los trabajadores, han sido trabajados en menor medida. En la actualidad, la literatura precedente ha confirmado la relevancia de los aspectos intrínsecos del trabajo como la autonomía, su utilidad social y las relaciones sociales para el bienestar de los trabajadores (Dahl, Nesheim y Olsen, 2009; Eurofound, 2014, 2016; Gallie, Felstead y Green, 2012; Green y Mostafa, 2012; Muñoz de Bustillo Llorente, Fernández Macías, Antón Pérez y Esteve Mora, 2011a). La aportación fundamental de esta investigación radica en el estudio del impacto de la estratificación social y laboral en la calidad intrínseca del trabajo. Para ello se ha construido un Índice de Calidad Intrínseca del Trabajo que aborda los aspectos fundamentales que intervienen en el desarrollo de la actividad laboral. Hasta la fecha, los estudios de estratificación social han distinguido dos lógicas laborales en el contexto de las transformaciones de los sistemas de producción en las sociedades avanzadas (EspingAndersen, 1993). La lógica laboral se define por el tipo de trabajo, su configuración y organización, la prevalencia de relaciones de autoridad, y la cualificación requerida (Hertel, 2017; Oesch, 2006). Por un lado, encontramos una lógica de tipo industrial caracterizada por una orientación organizacional/administrativa, con una estricta jerarquía de mando, y formas de organización laboral fordista. Y por otro, una lógica post-industrial que se caracteriza por una orientación al cliente y/o a la comunidad científica, formas de autoridad más igualitarias y organización del trabajo basada mayoritariamente en formas atípicas de empleo. Esto implica que las posiciones sociales no solo tienen una dimensión vertical sino también horizontal. Dicho de otra forma, los procesos de estratificación no solo se producen de arriba abajo sino de forma relacional.

Para abordar este campo de estudio vamos a establecer siete secciones. La primera parte revisa las aportaciones teóricas de las lógicas de empleo. La segunda parte hace lo mismo con la calidad intrínseca del trabajo. En la tercera se construyen las hipótesis con las que vinculamos la calidad laboral intrínseca con las lógicas de empleo en las que se enmarcan los trabajadores. La cuarta describe las fuentes, variables y metodología que vamos a emplear. Es decir, tratamos la operacionalización de los constructos teóricos. En la quinta se establecen, mediante análisis estadístico, la vinculación entre la calidad laboral intrínseca y las distintas lógicas resultantes. Esto es, analizamos la estratificación social de la calidad intrínseca en función de las lógicas laborales. En último lugar presentamos las principales conclusiones.

Los resultados que hemos obtenido apuntan a diferencias significativas de la calidad intrínseca del trabajo en función de los diferentes modelos obtenidos. En primer lugar descubrimos cinco lógicas laborales: Baja Industrial, Baja Post-industrial, Mixta, Profesional e Independiente. Éstas son resultado de diferencias de tipo vertical y horizontal. En segundo lugar hemos obtenido un índice compuesto que mide la calidad intrínseca del trabajo. Se trata del Índice de Calidad Intrínseca del Trabajo, que recoge las siguientes dimensiones: Autonomía, Interacción, Intensidad y Sentido. Para su operacionalización, nos hemos centrado en los indicadores que miden exclusivamente los aspectos que la literatura ha considerado más relevantes en torno a las características propias del desempeño de la actividad laboral. Para su construcción y validación hemos realizado análisis de factor común y factorial confirmatorio. 
En tercer lugar, hemos realizado un análisis de regresión lineal múltiple, que ha presentado el efecto que tienen las diferentes lógicas laborales sobre la calidad intrínseca. El efecto ha resultado significativo estadísticamente, lo que indica que la calidad intrínseca del trabajo difiere en función de las distintas lógicas de empleo en las que se ubiquen los trabajadores, tanto a nivel vertical como horizontal. A nuestro juicio, este resultado supone una importante aportación en el ámbito de la estratificación social y revela un nuevo tipo de desigualdad estructural respecto a la calidad intrínseca del trabajo: el acceso diferenciado a los aspectos de mayor relevancia en el desarrollo de la actividad laboral. La posibilidad de los trabajadores de ejercer autonomía, la mayor o menor intensidad en el desarrollo de la actividad, la viabilidad de interacciones de calidad en el trabajo, y el tener un sentido de utilidad social y de reconocimiento por el trabajo realizado se ven influenciados en función de las lógicas de empleo en las que se ubiquen los trabajadores, así que éstas otorgan beneficios intrínsecos diferenciados.

\section{LAS LÓGICAS DE EMPLEO EN LAS SOCIEDADES AVANZADAS}

Las teorías de la segmentación del mercado de trabajo (Doeringer y Piore, 1983; Piore, 1983) fueron las primeras en cuestionar la existencia de un mercado homogéneo, al señalar que la calidad laboral estaría sujeta a la estratificación del mercado en dos tipos: un segmento primario (independiente y dependiente) con una alta calidad laboral, y un segmento secundario con una baja calidad laboral. Los autores establecen así una diferenciación vertical de la calidad laboral entre las ocupaciones de ambos segmentos. Esta tipología ha resultado muy útil para entender la estratificación ocupacional en la etapa industrial, al demostrar la vinculación entre las ocupaciones y la calidad del trabajo en base a segmentos diferenciados, pero las transformaciones producidas en el modelo productivo y en las formas de organización laboral requieren ampliar el enfoque "Estos esquemas y las interpretaciones que les acompañan se muestran insuficientes para recoger los cambios que experimentan los mercados de trabajo en años más recientes" (Alós Moner, 2008, p. 128).

Como es bien sabido, en la década de los ochenta y noventa se producen una serie de transformaciones en los mercados laborales, cambios en la estructura productiva y en las estrategias que adoptan las empresas en cuanto a modelos organizativos, y cambios de índole social como el aumento en el nivel educativo de la población o la incorporación de la mujer al mercado laboral con voluntad de permanencia (Alós Moner y Jódar, 2005). Estas transformaciones dan lugar a un nuevo tipo de segmentación laboral de tipo horizontal, en base a dos modelos productivos diferenciados. En primer lugar, el modelo de producción industrial caracterizado por un tipo organización fordista, con una fuerte división del trabajo, una estructura jerárquica piramidal, y una centralización de las responsabilidades. En segundo lugar, un modelo de producción de economía de servicios, con un tipo de organización post-fordista caracterizado por estructuras de autoridad más horizontales, equipos auto-responsables, una mayor participación de los trabajadores en la toma de decisiones en los niveles inferiores y mayores activos específicos (Bauer, 2004; Hertel, 2017). Bell (1973) había denominado a este nuevo modelo como "sociedad post-industrial", ya que el foco se centra en el control del conocimiento científico que mejoraría la calidad en el empleo de los trabajadores.

El paso de un modelo de sociedad industrial a otra post-industrial ha generado cambios en las formas de organización del trabajo, en las formas de estratificación social y en las lógicas de empleo (Hertel, 2017; Oesch, 2006; Requena y Stanek, 2015). Entendemos por lógicas de empleo el tipo de organización del trabajo, los ajustes del proceso laboral, la 
prevalencia de relaciones de autoridad y las cualificaciones requeridas para la realización de las tareas. Las lógicas laborales están inevitablemente vinculadas al contexto y las experiencias que los individuos tienen cada día en el trabajo (Hertel, 2017; Oesch, 2006). La transformación hacia modelos de organización post-industrial ha supuesto la configuración de nuevas lógicas laborales ya que, según algunos autores, el trasvase desde la manufactura hacia modelos de trabajo basados en la economía del conocimiento supondría una mejora de la autonomía y la autoridad de los trabajadores, y el declive de las jerarquías fordistas (Esping-Andersen, 1992; Hertel, 2017; Piore y Sabel, 1990).

En este contexto de mayor complejidad de los mercados laborales, el esquema que presentamos tienen en cuenta la segmentación vertical propia de la teoría del mercado dual, pero introduce un tipo de segmentación horizontal, basada en la diferenciación entre los dos tipos de lógicas laborales, que responden a la esfera industrial y a la postindustrial. Estas lógicas comprenden aspectos claves tales como la incorporación de la mujer al mercado laboral, la menor dimensión de muchas empresas, la temporalidad o el grado de supervisión que tienen los trabajadores (Hertel, 2017), características que nos van a permitir configurar el esquema de lógicas laborales y su vinculación con la calidad laboral. En el siguiente epígrafe pasamos a describir las características que configuran tales lógicas laborales.

\section{Lógica organizacional vs Interpersonal}

La literatura que ha analizado las lógicas de empleo en el marco de la economía industrial y post-industrial ha planteado una tipología basada, por un lado, en una lógica administrativa/organizacional y por otro, en una lógica interpersonal.

La primera lógica de empleo es la típica del modelo fordista, tiene una orientación hacia la organización y un escenario de trabajo burocrático. Se establece una jerarquía de mando bien definida, siempre respaldada por la organización que otorga poder sobre otros (Hertel, 2017; Oesch, 2006). En este modelo, la pericia técnica está subordinada a la autoridad organizacional, que es de importancia superlativa dada la integración vertical de las tareas y las condiciones laborales (sujetas a parámetros bien definidos).

La autoridad es el eje que marca las posiciones de clase y las relaciones de empleo. "La omnipresencia de las relaciones de autoridad que caracterizan las ocupaciones industriales como posiciones que solo existen en referencia a la estructura jerárquica de los procesos de trabajo" (Hertel, 2017, p. 94).

El modelo postindustrial, en cambio, se basa en una lógica de trabajo interpersonal, caracterizada por una fuerte orientación hacia el cliente y/o a la comunidad profesional, las interacciones personales y el servicio cara a cara (Oesch, 2006). Esta fuerte relación interpersonal hace que las habilidades sociales y la cualificación sean importantes activos para conseguir exitosas interacciones cara a cara con los clientes y colegas. En este modelo la estructura de mando existe pero es sin duda más difusa, ya que las ocupaciones cuentan con un mayor grado de autonomía en relación al proceso de trabajo (Esping-Andersen, 1993, 1999). La pericia es el activo más destacado, por encima de la capacidad de monitorear el trabajo del modelo industrial. Esto es porque el acceso a las posiciones de clase depende de la posesión de credenciales educativas. "La organización laboral y el proceso laboral en las ocupaciones post industriales están relacionadas con la pericia individual y la agencia, más que la actuación estrictamente bajo órdenes" (Hertel, 2017, p. 95).

Las lógicas laborales forman parte del contexto en el que los trabajadores desarrollan su actividad, por lo que el escenario de trabajo es otra dimensión que las diferencia. En el modelo industrial el escenario de trabajo se enmarca en el formato de gran empresa, en cambio en el modelo post-industrial el tamaño de empresa se ha reducido (Hertel, 2017). 
La estructura de autoridad predominante en las organizaciones también marca la diferencia entre ambos modelos. Las ocupaciones en el modelo post-industrial se caracterizan por jerarquías más planas, mientras que las ocupaciones industriales forman parte de una clara cadena de mando (Esping-Andersen, 1993). Según Hertel (2017) las grandes empresas serían más propensas a la formación de jerarquías organizacionales, mientras que las empresas pequeñas, al depender más del cliente, tienden a favorecen una orientación más cercana. La afirmación de que la lógica de trabajo varía con el tamaño de la empresa fue apoyada por Oesch (2006), quien encuentra que las ocupaciones dentro de la lógica del servicio interpersonal son más frecuentes en empresas más pequeñas, mientras que la lógica de trabajo organizacional se da mayormente en grandes empresas.

El modelo industrial y el post-industrial difieren también respecto al tipo de organización del trabajo, lo que se plasma en el tipo de contrato. El contrato de trabajo estándar (en la línea con el modelo de varón breadwinner) es característico del modelo industrial fordista, mientras que el tipo de contrato temporal y el trabajo a media jornada son más característicos de las ocupaciones del modelo post-fordista (Hertel, 2017; Kalleberg, 2000).

Aunque la temporalidad hoy en día es transversal a las ocupaciones, también es cierto que es una de las características distintivas del modelo de organización post-industrial, con mayor incidencia entre las ocupaciones de servicio poco cualificado (Polavieja, 2003).

Para finalizar, la última dimensión que establece las diferencias horizontales entre ambos modelos es el género. La participación de las mujeres en la actividad laboral es un indicador que diferencia ambas lógicas laborales. "Uno de los factores que iniciaron la post-industrialización en sí misma es la mercantilización de los cuidados y de los servicios interpersonales, los cuales habían sido, en la era del fordismo, tradicionalmente producidos por el ama de casa" (Hertel, 2017, p. 122). El crecimiento del sector servicios en el modelo post-industrial está muy vinculado a la incorporación de la mujer al mercado laboral y a lo que algunos autores han denominado la "feminización" de las ocupaciones en el sector servicios (Esping-Andersen, 1993, 1999; Hertel, 2017; Hochschild, 1979; Santos Ortega, 2012). Por el contrario, en las ocupaciones más ligadas al sector industrial la participación de la mujer es muy inferior a la de los hombres.

Para concluir, las lógicas laborales se enmarcan en el contexto en el que los trabajadores desarrollan su actividad y plasman las diferencias tanto horizontales como verticales entre las ocupaciones en ambos modelos. Las dimensiones que marcan las principales diferencias son: la orientación primaria, hacia la organización o hacia el cliente; el escenario de trabajo, ubicado en la gran empresa o en tamaño reducido; el tipo de autoridad, basada en estructuras jerárquicas o mas horizontales; la distribución de la educación y el requerimiento de credenciales; el tipo de contratación, basada en el modelo estándar o formas atípicas de empleo; y la mayor o menor participación de la mujer en ambos modelos.

\section{Dos escenarios para las clases sociales}

En el modelo industrial, las clases sociales se definen en función de la jerarquía de mando y la autoridad. Las relaciones de empleo reflejan el principio de dominación dentro de la relación empleador y empleado y la lógica del trabajo es administrativa y organizacional. En cambio, en el modelo post-industrial las ocupaciones están vinculadas al sector servicios y se establece un modelo de relaciones laborales interpersonales. Las clases se configuran en función de la cualificación y los activos específicos. En este modelo, la distribución de la educación y las credenciales educativas marcan la diferencia principal entre las clases. Los requerimientos de habilidades, ya sean sociales (interpersonales) o 
académicas (credenciales), juegan un papel más importante en la posición vertical de clase que la capacidad de monitorear o supervisar (Hertel, 2017).

En este sentido, Esping-Andersen (1999) establece una tipología de clases sociales basada en la división del trabajo entre el modelo industrial y el post-industrial. Las diferencias se conforman en base a tres características: el grado de autoridad, de responsabilidad y el nivel de capital humano (Esping-Andersen, 1993). El autor enfatiza la importancia de la autonomía en el lugar de trabajo, la cual difiere en función de los respectivos tipos de la división del trabajo (Esping-Andersen, 1999). Mientras que las ocupaciones fordistas tienden a ser localizadas en una posición exacta en una estricta cadena de mando que limita la autonomía en el trabajo, en la organización post-industrial esta jerarquía es más borrosa e indefinida. Asimismo, las ocupaciones tienden a ser caracterizadas por un mayor grado de autonomía. Por su parte, Oesch (2006) distingue cuatro lógicas de empleo: independiente, técnica, organizativa e interpersonal. La primera de ellas se correspondería con las dinámicas propias del propietario, la segunda y tercera con las dinámicas industriales y la cuarta con lógicas post-industriales.

\section{Operacionalización de las lógicas de empleo}

A continuación, bosquejamos las características específicas de cada lógica de empleo. Lo hacemos de una forma que nos permita recoger datos empíricos y validar nuestras hipótesis.

En primer lugar, el ámbito en el que trabajan los individuos o, dicho de otra manera, el escenario donde se ubican los trabajadores en el desarrollo de su actividad, es un distintivo de ambos modelos. Las ocupaciones que trabajan en grandes empresas están más caracterizadas por una lógica de trabajo jerárquica y organizacional, mientras que las empresas pequeñas están más enfocadas al cliente, por lo que la lógica de trabajo es más interpersonal, como hemos señalado más arriba. La lógica de trabajo varía con el tamaño de la empresa (Oesch, 2006), lo que permite una de las distinciones de ambos modelos. Empleamos como indicadores el tamaño de la empresa y el porcentaje de trabajadores en empleo público como proxy de la prevalencia de las lógicas laborales (Hertel, 2017).

En segundo lugar, la estructura de autoridad es otro distintivo de las diferencias en ambos modelos. Según Esping-Andersen (1993), el modelo industrial se caracteriza por una clara cadena de mando, mientras que en el modelo post-industrial la estructura de dirección es mas plana. Para analizar esta dimensión se analiza tanto la prevalencia del supervisor como la de ser supervisado. Se espera que una mayor frecuencia en la supervisión de los trabajadores se corresponda en mayor medida con el modelo industrial.

En tercer lugar, otra dimensión que distingue a ambos modelos es el tipo de organización del trabajo. El modelo industrial se caracteriza por un tipo de contrato estándar propio del modelo fordista, mientras que el modelo post-industrial se caracteriza por formas atípicas de empleo. El tipo de contrato temporal y la jornada parcial son los indicadores empleados para distinguir las lógicas laborales en ambos modelos. Según estudios previos, cabe esperar que las condiciones atípicas de empleo sean más probables en el modelo postindustrial (Kalleberg, 2000).

En cuarto lugar, el género es una variable distintiva de ambos modelos, ya que la incorporación de la mujer de manera permanente al mercado laboral está muy vinculada al crecimiento del sector servicios (Hertel, 2017). La participación de la mujer es el indicador usado, se espera que en el modelo post-industrial se dé una mayor participación que en el industrial, especialmente en las ocupaciones relacionadas con las clases trabajadoras de servicio. 
En quinto lugar, otro de los indicadores empleados se corresponde con los beneficios adicionales obtenidos en el desempeño de la actividad laboral. En el modelo industrial esta clase de beneficios adicionales es menos común, en el modelo post-industrial se dan con mayor frecuencia.

Por último, los activos específicos, las denominadas credenciales educativas, es otra de las dimensiones fundamentales que distinguen las lógicas de empleo de ambos modelos. Las sociedades post-industriales se caracterizarían en mayor medida por trabajos que requieren cualificación como requisito de acceso (Hertel, 2017; Kalleberg, 2011). El indicador empleado para medir los activos específicos es el nivel de estudios.

En la Tabla 1 se presentan las dimensiones que contiene cada lógica laboral, sus respectivos indicadores y la expectativa sobre su prevalencia en cada modelo.

Tabla 1. Dimensiones e indicadores de las lógicas del trabajo industrial y post-industrial

\begin{tabular}{|c|c|c|c|}
\hline Dimensión & Indicadores & Industrial & Post-industrial \\
\hline \multirow{3}{*}{$\begin{array}{l}\text { Ámbito } \\
\text { laboral }\end{array}$} & Servicio público & Bajo & Alto \\
\hline & & & \\
\hline & $\begin{array}{c}\text { Tamaño de la } \\
\text { empresa }\end{array}$ & Alto & Bajo \\
\hline \multirow[t]{2}{*}{$\begin{array}{l}\text { Estructura de } \\
\text { autoridad }\end{array}$} & $\begin{array}{l}\text { Estatus del } \\
\text { supervisor }\end{array}$ & Alto & Bajo \\
\hline & Estatus del control & Alto & Bajo \\
\hline \multirow{2}{*}{$\begin{array}{l}\text { Organización } \\
\text { del trabajo }\end{array}$} & Contrato temporal & Bajo & Alto \\
\hline & Jornada parcial & Bajo & Alto \\
\hline Género & Mujer & Bajo & Alto \\
\hline Remuneración & $\begin{array}{l}\text { Beneficios } \\
\text { adicionales }\end{array}$ & Bajo & Alto \\
\hline $\begin{array}{c}\text { Activos } \\
\text { específicos }\end{array}$ & $\begin{array}{c}\text { Educación } \\
\text { Post-Obligatoria }\end{array}$ & Bajo & Alto \\
\hline
\end{tabular}

Fuente: elaboración propia a partir de Hertel (2017)

\section{LA CALIDAD INTRÍNSECA DEL TRABAJO}

La calidad del trabajo ha sido una de las principales preocupaciones de los científicos sociales, lo que se manifiesta en la amplia literatura que aborda la construcción de modelos que miden esta dimensión fundamental en las sociedades avanzadas (Anker, Chernyshev, Egger, Mehran y Ritter, 2003; Bericat y Cascales Mira, 2019; Bescond, Châtaignier y Mehran, 2003; Bonnet, Figueiredo y Standing, 2003; Davoine, Erhel y Guergoat, 2008a; Ghai, 2002; Green y Mostafa, 2012; Leschke y Watt, 2008; Tangian, 2007, entre otros). Pero a pesar de 
la creciente preocupación entre investigadores e instituciones, no existe una definición estándar sobre la calidad laboral en la literatura académica. La mayoría de autores coincide en que es un concepto multidimensional (Dahl et al., 2009; Davoine et al., 2008a; Fernández-Macías, 2012; Kalleberg, 2011; Martel y Dupuis, 2006; Royuela, López-Tamayo y Suriñach, 2008), pero no todos coinciden en cuáles son las dimensiones a analizar (Davoine, Erhel y Guergoat, 2008b). Es por ello que el desarrollo de un simple indicador es claramente insuficiente $y$, en consecuencia, se hace necesario acudir a un enfoque de indicadores compuestos para abarcar su enorme complejidad.

La literatura económica ha empleado indicadores monetarios para analizar si un trabajo es de calidad o no, basándose sobre todo en el nivel de ingresos. Aunque se da una relación lineal entre ingresos y satisfacción el efecto sobre esta última disminuye hasta cierto punto con el aumento de los ingresos. Es lo que se denomina la paradoja de la felicidadingresos (Easterlin, 1974). Asimismo, un incremento en el salario no implica una mejora en aspectos claves para el bienestar del trabajador como la autonomía en el desempeño de la actividad, el esfuerzo realizado y la intensidad de las demandas (Green, 2006). La calidad del trabajo, entendida desde una perspectiva más amplia, comprende no solo el salario sino otras condiciones de empleo que han recibido menor atención (Muñoz de Bustillo Llorente, Fernández Macías, Antón y Esteve, 2009). Algunos trabajos pueden contar con un sueldo relativamente modesto y aún así ser considerados trabajos de calidad, porque son significativos, permiten que el individuo pueda ejercer autonomía y favorecen interacciones sociales de calidad.

La perspectiva sociológica ha resaltado la importancia de los aspectos intrínsecos frente a los extrínsecos o monetarios en el análisis de la calidad laboral (Dahl et al., 2009; Kalleberg, 2011; Green 2006; Green y Mostafa, 2012; Muñoz de Bustillo Llorente, Fernández Macías, Esteve Mora y Antón, 2011b). Las características del trabajo consideradas «intrínsecas» son las que se centran en la naturaleza de la propia actividad, aquí la recompensa es derivada de la experiencia del trabajo en sí mismo, mientras que las «extrínsecas» describen las consecuencias del trabajo (salario, ascenso, prestigio) o las condiciones bajo las cuales se realiza (Seeman, 1967). Las primeras son un fin en sí mismas, mientras las segundas son un medio para conseguir un fin mayor, alcanzar el bienestar. En términos de motivación, las recompensas intrínsecas están basadas en el desarrollo de la actividad en sí, desde una lógica expresiva del yo, frente a la lógica instrumental basada en el resultado, que caracteriza a las condiciones extrínsecas del trabajo. Un trabajo con recompensas intrínsecas es normalmente interesante y desafiante, uno es capaz de usar las destrezas y habilidades, es capaz de tener discreción en su trabajo y aprender cosas nuevas, tener autonomía y ser reconocido por hacer un trabajo bien hecho (Kalleberg y Vaisey, 2005). Estudios previos muestran que las características intrínsecas se relacionan más consistentemente con la satisfacción en el trabajo que las recompensas extrínsecas (Cascales Mira, 2010; Kalleberg, 1977). “Los trabajadores que son capaces de controlar cómo y qué hacen en el trabajo tienen mayor probabilidad de obtener recompensas intrínsecas de sus trabajos. Las recompensas intrínsecas son beneficios y utilidades que la gente obtiene de la realización de las tareas, como opuesto a recompensas extrínsecas como dinero o beneficios extra que la gente obtiene por el desarrollo de su trabajo" (Kalleberg, 2011, p. 7).

Con el cambio en la naturaleza del trabajo hacia modelos post-industriales, la orientación intrínseca ha adquirido una mayor importancia en los estudios de calidad laboral, también como un factor de competitividad en las sociedades basadas en la economía del conocimiento y la información (Gallie, 2007). Los trabajos se han convertido en más significativos, permitiendo más oportunidades de auto-realización, equipos auto-dirigidos con una mayor autonomía y menor esfuerzo físico (Dahl et al., 2009). De los aspectos de calidad laboral las características intrínsecas son las que tienen el mayor impacto en el 
bienestar, ya que a medida que la calidad en el trabajo se deteriora, la variabilidad en el bienestar aumenta (Eurofound, 2012b). Es por ello que los componentes intrínsecos del trabajo están siendo incorporados de manera creciente en los estudios de calidad laboral (Dahl et al., 2009; Eurofound, 2012a, 2014, 2016; Gallie et al., 2012; Green y Mostafa, 2012).

Tras la revisión de las características intrínsecas que los autores han considerado componentes clave para analizar la calidad laboral, hemos elaborado un Índice de Calidad Intrínseca del Trabajo compuesto por cuatro dimensiones: la capacidad de discreción de los trabajadores sobre su trabajo (Autonomía), el entorno social en el que los trabajadores interactúan (Interacción), la intensidad en términos de demandas físicas y psicológicas con las que se enfrentan los trabajadores en el puesto de trabajo (Intensidad) y el sentido o utilidad social del trabajo realizado (Sentido).

\section{LA CALIDAD INTRÍNSECA DEL TRABAJO EN EL MARCO DE LAS LÓGICAS DE EMPLEO}

Las lógicas laborales forman parte del contexto en el que se enmarcan los trabajadores y configuran muchos aspectos de sus condiciones laborales: la prevalencia de criterios de autoridad y supervisión frente a criterios de cualificación como ejes de estratificación ocupacional; la posibilidad de una mayor o menor participación y control por parte del trabajador en el desempeño de su actividad laboral; o el predominio de un modelo organizacional basado en relaciones jerárquicas o por el contrario basado en relaciones horizontales (Hertel, 2017). En este sentido, las lógicas laborales tienen un impacto en la calidad intrínseca del trabajo, pero la dirección del impacto varía en función de la perspectiva.

Por un lado, las investigaciones derivadas de la Critical Management Studies (Fernández Rodríguez, 2007, 2016; Fernández Rodríguez y Medina-Vicent, 2017; Lahera Sánchez, 2004; Pérez Zapata, Álvarez Hernández y Castaño Collado, 2017; Pérez-Zapata, Pascual, AlvarezHernández y Collado, 2016) denuncian la creciente intensificación del trabajo y la exigencia de identificación del trabajador con los objetivos de la empresa, como parte de la nueva cultura empresarial post-fordista (Pérez Zapata et al., 2017). A su vez, características distintivas del modelo como la autonomía o la mayor interacción de los trabajadores, a través del trabajo en equipo, son vistas como una nueva forma de control por parte de la organización, mostrando la cara "oscura" de la nueva cultura organizacional (Alonso y Fernández Rodríguez, 2013; Fernández Rodríguez, 2016).

Por otro lado, diversos estudios muestran que el apoyo social y el entorno organizacional, así como la participación y la autonomía en el trabajo son, en la actualidad, factores clave para el bienestar de los trabajadores (Eurofound, 2016; Gallie, Felstead, Green e Inanc, 2014; Gallie, Zhou, Felstead, Green y Henseke, 2017; Green, 2006; Green y Mostafa, 2012; Kalleberg, 2011) y destacan el "valor inherente a los empleados de poder ejercer control sobre sus vidas laborales, ya sea por necesidades básicas de autonomía o por valores culturales sobresalientes de autodeterminación" (Gallie et al., 2017, p. 187).

En este marco queremos analizar si la calidad intrínseca del trabajo varía en función de las distintas lógicas laborales y, si lo hace, en qué dirección. Para ello hemos partido de una serie de hipótesis que presentamos en el siguiente epígrafe.

\footnotetext{
1 Critical Management Studies es una corriente alternativa a las teorías organizacionales ortodoxas. Desde finales de la década de los ochenta, visibiliza elementos importantes en las nuevas realidades organizacionales como son la subjetividad, la identidad, el conflicto, el poder o las técnicas de control gerenciales.
} 


\section{LAS HIPÓTESIS. VINCULACIÓN ENTRE LAS LÓGICAS DE EMPLEO Y LA CALIDAD INTRÍNSECA DEL TRABAJO}

Tomando en cuenta la importancia de los componentes intrínsecos de la actividad laboral en las sociedades contemporáneas, nuestra intención es averiguar si la calidad intrínseca del trabajo varía efectivamente en función de las lógicas de empleo. Se espera que las sociedades industriales, al caracterizarse por una lógica de empleo con una rígida jerarquía de mando, la mayor estandarización de las actividades en el empleo y una menor posibilidad de las relaciones interpersonales, tengan una puntuación inferior en el índice de calidad intrínseca.

Por el contrario, en las sociedades post-industriales el desempeño de la actividad está muy vinculado con el desarrollo de las relaciones inter-personales, empleos menos reiterativos en los que el conocimiento tiene un papel central y los trabajadores están menos supervisados y disponen de una mayor autonomía, por lo que se espera que disfruten de una mayor calidad intrínseca del trabajo. En este sentido, partimos del supuesto de que los trabajos que disponen de buenas condiciones laborales atienden no solo a las diferencias basadas en el salario o las relaciones de empleo, propias del modelo industrial, sino también a las diferencias basadas en los componentes intrínsecos del desarrollo de la actividad como las relaciones entre los trabajadores, el sentido en el trabajo realizado o la posibilidad de ejercer mayor autonomía.

Por último, en relación a la configuración de las lógicas de empleo, esperamos que el análisis de clases latente resultante defina más de dos modelos, es decir, esperamos que se extraigan lógicas de empleo heterogéneas, ya que las lógicas laborales no solo están segmentadas verticalmente sino también horizontalmente.

\section{FUENTES, VARIABLES Y ESTRATÉGIA METODOLÓGICA}

Para relacionar las distintas lógicas laborales con la calidad intrínseca del trabajo vamos a emplear la European Working Conditions Survey (EWCS, en sus siglas en inglés) la oleada de 2015. La Fundación europea para la mejora de vida en el trabajo (Eurofound, en sus siglas en inglés) es la institución encargada de llevar a cabo esta encuesta. Para España, disponemos de 3154 unidades. Hemos filtrado la muestra a 3139 ya que se han seleccionado sólo a los individuos de más de 30 años (aquellos que hayan adquirido la madurez (aboral) y de menos de 65 años.

No ha sido difícil encontrar las variables necesarias para llevar a cabo nuestra investigación, ya que en la línea de Muñoz de Bustillo y colegas (2011a, 2011b) la EWCS es una de las encuestas más completas y exhaustiva sobre condiciones de trabajo. Esta encuesta tiene una amplia batería de preguntas relativas a los aspectos intrínsecos del trabajo y a las lógicas laborales. Basándonos en la teoría bosquejada más arriba, hemos contado con las siguientes variables que pasamos a comentar.

El modelo de medición del Índice de Calidad Intrínseca del Trabajo (ICIT) se compone de cuatro dimensiones y 13 indicadores. Las dimensiones son: Autonomía, Interacción, Intensidad y Sentido. La tabla 2 presenta la información resumida de las dimensiones e indicadores que componen el índice. 
Tabla 2. Dimensiones e indicadores del Índice de Calidad Intrínseca del Trabajo

\begin{tabular}{|c|c|c|}
\hline Dimensión & Indicador & Variable \\
\hline \multirow{4}{*}{ Autonomía } & $\begin{array}{l}\text { Puede influir en las decisiones importantes } \\
\text { para tu trabajo }\end{array}$ & Q61n \\
\hline & Puede aplicar sus propias ideas en su trabajo & Q61i \\
\hline & $\begin{array}{c}\text { Es consultado antes de establecer los } \\
\text { objetivos }\end{array}$ & Q61C \\
\hline & Compañeros le apoyan & Q61a \\
\hline \multirow{3}{*}{ Interacción } & El jefe le apoya & Q61b \\
\hline & $\begin{array}{l}\text { Generalmente me llevo bien con los } \\
\text { compañeros }\end{array}$ & Q89d \\
\hline & Hay buena cooperación entre compañeros & Q70e \\
\hline \multirow{4}{*}{ Intensidad } & Trabajar a alta velocidad & Q49a \\
\hline & Trabajar con plazos ajustados & Q49b \\
\hline & Estar en posiciones cansadas o dolorosas & Q30a \\
\hline & Llevar o mover cargas pesadas & Q30C \\
\hline \multirow{2}{*}{ Sentido } & Hacer un trabajo útil & Q61j \\
\hline & Hacer un trabajo bien hecho & Q61h \\
\hline
\end{tabular}

Fuente: elaboración propia con datos de la EWCS (2015)

La dimensión Autonomía está compuesta por tres indicadores, que se corresponden con las siguientes variables:

"Para cada una de las siguientes afirmaciones, seleccione la respuesta que mejor describa su situación laboral:

Usted es consultado antes de que se establezcan objetivos para su trabajo,

Usted puede aplicar sus propias ideas en su trabajo, y

Usted puede influir en decisiones que son importantes para su trabajo", va desde 1 siempre a 5 nunca.

La siguiente dimensión es la Interacción y contiene un total de cuatro indicadores que se corresponden con las siguientes variables:

"Para cada una de las siguientes afirmaciones, seleccione la respuesta que mejor describa su situación laboral: Sus colegas le ayudan y apoyan y Su jefe le ayuda y apoya", va desde 1 siempre a 5 nunca;

“De la siguiente pregunta sobre su lugar de trabajo, ¿En qué medida está de acuerdo o en desacuerdo con las siguientes afirmaciones sobre su trabajo? En general, me llevo bien con mis compañeros de trabajo" que va de 1 muy de acuerdo a 5 muy en desacuerdo;

“En qué medida está de acuerdo o en desacuerdo con las siguientes afirmaciones? Hay buena cooperación entre usted y sus colegas", va de 1 muy de acuerdo a 5 muy en desacuerdo.

La tercera dimensión Intensidad (física y psicológica), está formada por 4 indicadores que se corresponden con las siguientes variables: 
"Por favor dígame, usando la misma escala, ¿su principal trabajo remunerado involucra ...?: Posiciones cansadas o dolorosas" y "llevar o mover cargas pesadas". Va de 1 todo el tiempo a 7 nunca.

Y "Su trabajo involucra: trabajar a una velocidad muy alta y trabajar con plazos ajustados". Va de 1 todo el tiempo a 7 nunca.

La cuarta dimensión es Sentido, y está formada por dos indicadores que se corresponden con las variables: "Tienes la sensación de hacer un trabajo útil" y "Su trabajo le da la sensación de trabajo bien hecho". Va de 1 siempre a 5 nunca.

Para construir las lógicas laborales nos hemos basado en los siguientes indicadores: tipo de empleo (público, privado), capacidad de supervisión (supervisa o no), cualidad de ser supervisado (si o no), tipo de jornada (completa o parcial), tipo de contrato (temporal o indefinido), género (hombre o mujer), tamaño de la empresa (de 0 a 9 empleados y más de 10 empleados), educación (obligatoria o menos, post-obligatoria o más) y pago por rendimiento (recibe otras prestaciones aparte del salario o no las recibe).

En relación a la estrategia metodológica, ésta ha consistido en tres pasos diferenciados. En el primero se ha construido el Índice de Calidad Intrínseca del Trabajo. En el segundo de ellos, aplicamos un análisis de clases latentes que extraiga las lógicas laborales predichas en el modelo teórico. Por último, mediante un análisis de regresión lineal verificamos la relación entre el índice y los grupos obtenidos.

El Índice de Calidad Intrínseca del Trabajo ha sido construido mediante un análisis factorial exploratorio, más concretamente, un análisis de factor común. Este índice ha sido validado empleando un análisis factorial confirmatorio, ya que es la técnica de análisis adecuada para comprobar la robustez del constructo (Bericat, 2018; Cea D'Ancona, 2002; Escobar, 2011). Por otro lado, los resultados obtenidos por el estadístico Alpha de Cronbach han permitido la validación interna de cada una de las dimensiones que componen el indice.

En relación al análisis de factor común, se ha utilizado el método de extracción de ejes principales ${ }^{2}$ con rotación varimax. Escogemos este tipo de rotación porque minimiza el número de variables con cargas altas en un factor, mejorando así la interpretación de los factores. Para la elección de los factores nos hemos basado en la regla de Kaiser, que utiliza el tamaño de los auto-valores como criterio para decidir el número de factores que estarán presentes en la solución factorial. Por último, el método de estimación de las puntuaciones factoriales ha sido el método de regresión ${ }^{3}$.

El análisis empleado para dilucidar las dos lógicas laborales ha sido un análisis de clases latentes. Ésta es una técnica clasificatoria que permite encontrar, mediante la estimación de probabilidades condicionadas, grupos subyacentes en un conjunto de datos (Magidson y Vermunt, 2004). Esto se hace gracias a la construcción de la variable latente categórica no observada. Asimismo, y esto es sin duda lo más importante, este análisis está dotado de criterios objetivos que guían la determinación del número de grupos (Kaufman y Rousseeuw, 1990). Podemos estimar los parámetros que nos den información sobre cuál es la cantidad de grupos más acertada. En concreto, como hemos señalado en las hipótesis, nuestra predicción sitúa al menos dos áreas laborales diferentes: una que representará la lógica industrial y otra la post-industrial.

La notación matemática del análisis de clases latentes es la siguiente (Monroy, Vidal y Saade, 2009). Siendo $\pi$ la probabilidad, $x_{t}$ la variable latente con $t$ categorías y $T$ el

2 El método de ejes principales es un método iterativo, es decir, un método que se ejecuta repetitivamente hasta alcanzar la solución idónea.

3 Utiliza el método de los mínimos cuadrados para estimar las puntuaciones. Este método da lugar a puntuaciones con máxima correlación con las puntuaciones teóricas. 
total de casos de la variable latente. Entonces, $\pi\left(x_{t}\right)$ es la probabilidad que un individuo seleccionado aleatoriamente pertenezca a la clase latente $t(t=1,2, \ldots, T)$ y $\pi\left(y_{i} \mid x_{t}\right)$ es la probabilidad de un individuo de tener el valor i en la variable y dada la pertenecía a la clase t en la variable $x$.

En consecuencia, $\pi\left(y_{1} y_{2} y_{3} y_{4} \mid x_{t}\right)$ es la probabilidad conjunta de una serie de valores de respuesta dada la pertenecía a la clase t en la variable $\mathrm{x}_{\mathrm{i}}$.

Formalmente, el análisis de clases latentes más sencillo se expresa de la siguiente manera.

$$
\pi(Y i \mid y)=\sum_{t=1}^{T} \pi\left(x_{i}=t\right) \prod_{j=1}^{J} \pi\left(Y_{i j}=y_{i j} \mid X_{i}=t\right)
$$

Donde $Y$ es variable manifiesta para el caso $\mathrm{i}, \mathrm{Y}_{\mathrm{ij}}$ la variable manifiesta para el caso $\mathrm{i}$ en la variable $\mathrm{j}$, con J número de variables en el modelo y $\mathrm{X}_{\mathrm{i}}$ variable latente $\mathrm{t}$ que indica una clase latente particular con T números de clases latentes.

Los ajustes estadísticos empleados en el modelo de clases latentes son el Criterio de Información Bayesiana (BIC) y el Criterio de Información de Aikaike (AIC). Se expresa de la siguiente manera:

$$
\begin{aligned}
& B I C=2(\log ) L+q(\log ) N \\
& A I C=2(\log ) L+2 q
\end{aligned}
$$

En las que q es el número de parámetros del modelo y $N$ es el número total de unidades. El modelo que obtenga un BIC y AIC más bajo será el que presente un mejor ajuste estadístico.

Una vez calculado el Índice de Calidad Intrínseca del Trabajo y seleccionados los grupos que representan a cada lógica laboral, empleamos un análisis de regresión para comprobar la vinculación entre ambos. Se expresa con la siguiente ecuación.

$$
Y=\alpha+X_{k}+X_{c}+\varepsilon
$$

Donde $Y$ es la puntuación en el Índice de Calidad Intrínseca del Trabajo, a es el intercepto, $X_{k}$ representa cada una de las clases latentes obtenidas en el análisis previo, $\mathrm{y}_{\mathrm{c}}$ las variables de control que, en este caso, es la edad del individuo. Por último, $\varepsilon$ es el error.

\section{RESULTADOS}

\section{Primer paso: Construcción y validación del Índice de Calidad Intrínseca del Trabajo.}

En este epígrafe, vamos a comprobar la validez y robustez del índice de Calidad Intrínseca del Trabajo. El resultado de las iteraciones del análisis de factor común ha sido la obtención de 4 dimensiones o factores latentes que componen el ICIT. Los cuatro factores 
explican el 61,9\% de la varianza, con un $\mathrm{KMO}^{4}$ de 0,765. La medida de adecuación muestral KMO (Kaiser-Meyer-Olkin) contrasta las correlaciones parciales entre las variables. Según la literatura especializada, un valor superior a 0,75 es un buen valor, por lo que se confirma la adecuación de la muestra a la hipótesis del modelo como buena. Los indicadores han obtenido puntuaciones altas en cada factor, por lo que podemos decir que representan bien el contenido de cada dimensión latente.

La Tabla 3 presenta los pesos factoriales de la matriz rotada. Una alta puntuación del indicador implica una mayor contribución al contenido de esa dimensión.

Tabla 3. Pesos factoriales de los indicadores para cada uno de los factores

\begin{tabular}{|c|c|c|c|c|}
\hline \multirow[t]{2}{*}{ Indicadores intrínsecos } & \multicolumn{4}{|c|}{ Pesos factoriales } \\
\hline & $\begin{array}{c}\text { F1 } \\
\text { Autonomía }\end{array}$ & $\begin{array}{c}\text { F2 } \\
\text { Interacción }\end{array}$ & $\begin{array}{c}\text { F3 } \\
\text { Intensidad }\end{array}$ & $\begin{array}{c}\text { F4 } \\
\text { Sentido }\end{array}$ \\
\hline Compañeros le apoyan & 169 & ,751 &,- 006 & ,077 \\
\hline Jefe le apoya & ,302 & ,577 & ,091 & ,098 \\
\hline $\begin{array}{l}\text { Buenos amigos/ } \\
\text { compañeros }\end{array}$ & ,052 &, 490 & ,056 &, 342 \\
\hline Buena cooperación & 082 & ,557 & ,069 & ,337 \\
\hline Decisiones importantes &, 754 & ,112 & ,064 & ,148 \\
\hline $\begin{array}{c}\text { Aplicar las propias } \\
\text { ideas }\end{array}$ &, 660 & ,147 & ,099 & ,220 \\
\hline Es consultado & ,613 & 209 & 105 & ,090 \\
\hline Trabajar a alta velocidad &,- 001 & ,007 & 805 & ,073 \\
\hline $\begin{array}{c}\text { Trabajar con plazos } \\
\text { ajustados }\end{array}$ &,- 077 & ,024 & ,702 & ,067 \\
\hline $\begin{array}{c}\text { Trabaja con posiciones } \\
\text { dolorosas }\end{array}$ & 163 & ,061 &, 456 &,- 003 \\
\hline $\begin{array}{l}\text { Llevar o mover cargas } \\
\text { pesadas }\end{array}$ & ,117 & ,038 & ,391 & ,020 \\
\hline $\begin{array}{l}\text { Realiza un trabajo útil } \\
\text { socialmente }\end{array}$ & ,205 & ,198 & ,055 &, 664 \\
\hline $\begin{array}{c}\text { Realiza un trabajo bien } \\
\text { hecho }\end{array}$ & ,203 &, 234 & ,063 & ,660 \\
\hline
\end{tabular}

Fuente: elaboración propia con datos de la EWCS (2015)

Si observamos en cada columna los pesos factoriales más altos (en negrita), podemos apreciar los indicadores intrínsecos que están más asociados a cada uno de los cuatro factores. La estructura de características intrínsecas de cada factor facilita su interpretación teórica, y nos permite ver qué aspectos constituyen los elementos clave de los factores latentes de calidad del trabajo. El procedimiento para interpretar el resultado es seleccionar los indicadores que saturan más intensamente cada factor, o que tienen los pesos factoriales más altos. De esta manera podemos seleccionar los indicadores más eficientes que representan cada dimensión de la calidad laboral. "Procediendo de esta

4 El índice de KMO nos da información sobre la adecuación de la muestra a las hipótesis del modelo de Análisis Factorial. 
manera avanzamos hacia la selección de un modelo al mismo tiempo válido y parsimonioso" (Bericat, 2018, p. 111). La estructura factorial ha quedado de la siguiente manera.

Los indicadores relativos a "poder tomar decisiones importantes en el trabajo", "poder aplicar las propias ideas" y "ser consultado antes de que se establezcan los objetivos" han concentrado la puntuación factorial más alta en torno al primer factor, que hemos etiquetado como la dimensión "Autonomía". Los indicadores que hacen referencia a la "buena cooperación en el trabajo", "las buenas relaciones con los compañeros", el "apoyo y ayuda del jefe" y el "apoyo y ayuda de los compañeros" han concentrado las puntuaciones factoriales más altas en el segundo factor, que hemos etiquetado como "Interacción". Los indicadores relacionados con las demandas psicológicas "trabajar a gran velocidad" y "trabajar con plazos ajustados" y las demandas físicas "trabajar en posiciones dolorosas" y "mover cargas pesadas" se han agrupado formando el factor latente que hemos denominado "Intensidad". Y, por último, los indicadores relativos a "realizar un trabajo útil" y "realizar un trabajo bien hecho" se han concentrado en el cuarto factor, formando la dimensión que hemos denominado "Sentido" en el trabajo.

Respecto a la puntuación de las comunalidades, es decir, la proporción de la varianza explicada por los factores comunes en una variable, en nuestro modelo los indicadores que más información aportan al factor son trabajar a alta velocidad $(0,654)$, poder tomar decisiones importantes $(0,607)$, los compañeros le apoyan $(0,598)$, realizar un trabajo bien hecho $(0,536)$ y realizar un trabajo útil $(0,525)$. Los indicadores relacionados con la intensidad y la autonomía son los que más información aportan al índice de calidad intrínseca. Los indicadores relacionados con la intensidad física son los que menos información aportan.

Para comprobar la fiabilidad interna de las escalas utilizadas para construir el índice, hemos aplicado el estadístico Alpha de Cronbach. La tabla 4 muestra los resultados de los ítems para cada dimensión. Las puntuaciones presentan una fiabilidad aceptable (Jisu, Delorme y Reid, 2006) 5 .

Tabla 4. Fiabilidad de escala de las dimensiones del Índice de Calidad Intrínseca del Trabajo. Alpha de Cronbach.

\begin{tabular}{ccc}
\hline Dimensiones & Ítems & Alpha de Cronbach \\
\hline Interacción & 4 & 0,67 \\
Autonomía & 3 & 0,76 \\
Intensidad & 4 & 0,69 \\
Sentido & 2 & 0,73 \\
Total & 13 & 0,71 \\
\hline
\end{tabular}

Fuente: Elaboración propia con datos de la EWCS (2015)

El siguiente análisis que hemos realizado tiene como objetivo validar la consistencia interna del modelo de medición. Para ello, hemos realizado un análisis factorial confirmatorio (AFC a partir de ahora) ya que la literatura especializada considera que es la técnica más apropiada para validar y confirmar este tipo de modelos (Bericat, 2014, 2018; Cea D'Ancona, 2002; Escobar, 2011). Los coeficientes estandarizados de regresión fueron estadísticamente significativos y las cargas factoriales obtuvieron valores superiores a 0,6 con lo cual puede señalarse que todos los indicadores saturan satisfactoriamente con sus respectivas variables latentes. La covarianza entre los factores no fue superior a 0,85 y por tanto no presentó problemas de colinealidad, lo que supone además una evidencia de su

5 El valor de fiabilidad en investigación exploratoria debe ser igual o mayor a 0,6; en estudios confirmatorios debe estar entre 0,7 y 0,8 . 
validez discriminante (Escobedo Portillo, Hernández Gómez, Estebané Ortega y Martínez Moreno, 2016) ${ }^{6}$.

En lo que se refiere al ajuste del modelo, los diversos indices de ajuste resultaron aceptables, por lo que se puede afirmar que el modelo propuesto acerca de la estructura factorial de la escala es sustentable. La Tabla 5 presenta los resultados de los test más utilizados para valorar la bondad de ajuste de los modelos.

Tabla 5. Índice de Calidad Intrínseca del Trabajo. Análisis factorial confirmatorio. Parámetros de bondad de ajuste del modelo.

\begin{tabular}{ccccccc}
\hline$p$ & GFI & AGFI & PNFI & NFI & TLI & RMSEA (IC 90\%) \\
\hline$<0,001$ & 0,936 & 0,902 & 0,666 & 0,901 & 0,843 & $0,084(0,083-0,086)$ \\
\hline
\end{tabular}

Fuente: elaboración propia con datos de la EWCS (2015)

\section{Segundo paso. Validación de las lógicas laborales}

Nuestra segunda estrategia consiste en validar las lógicas laborales. Para ello, como hemos señalado más arriba, hemos agrupado un conjunto de nueve variables capaces de discriminar entre la naturaleza industrial y post-industrial del trabajo.

Recordamos que lo que intentamos conseguir es una clasificación por grupos en función de las variables introducidas. Hemos comenzado nuestro análisis partiendo de una sola clase. Si no existiesen diferencias entre las variables introducidas sólo existiría una clase latente representada en un solo grupo. Dejaremos de ampliar las clases latentes una vez que el aumento de una clase latente no aporte una mejora de ningún ajuste estadístico. La tabla 6 presenta los resultados del análisis de clases latentes.

Tabla 6. Resultado del análisis de clases latentes.

\begin{tabular}{cccc}
\hline Número de clases latentes & BIC & AIC & Grados de libertad \\
\hline 1 clase & 23231.73 & 23179.23 & 9 \\
2 clases & 22643.56 & 22532.45 & 19 \\
3 clases & 22477.98 & 22308.34 & 29 \\
4 clases & 22425.45 & 22197.96 & 39 \\
$\mathbf{5}$ clases & $\mathbf{2 2 4 1 5 . 1 9}$ & $\mathbf{2 2 . 1 2 9 . 3 6}$ & $\mathbf{4 9}$ \\
\hline
\end{tabular}

Fuente: elaboración propia con datos de la EWCS (2015)

Como se puede apreciar en la Tabla 6 , el ajuste comienza a mejorar a medida que un mayor número de variables latentes son introducidas. Sin embargo, a partir de la clase latente número cinco, las diferencias dejan de ser significativas. Esto significa que cinco clases latentes son las adecuadas.

El primer grupo está representado por el $16 \%$ de los individuos de nuestra muestra. Estos individuos se caracterizan por ser mayormente mujeres (68\%) con educación básica

6 Tomando los criterios de Garson, Raubenheimer y Widaman en los que establecen que, para eliminar al máximo la colinealidad entre variables, es necesario tomar las variables cuyas cargas sean $\geq 0,07$ 
(91\%), que no supervisan (94\%) ni son muy supervisadas (60\%), que tienen media jornada (90\%) y empleos temporales (60\%). Trabajan para el sector privado $(90 \%)$ y para pequeñas empresas (78\%). Este primer grupo está formado, principalmente, por aquellos individuos que trabajan en el sector servicios en ocupaciones semi-cualificadas (21\%) o no cualificadas (33\%). En líneas generales, esta clase latente agrupa principalmente al sector de servicio con baja cualificación.

El segundo grupo está formado por el $25 \%$ de nuestra muestra. Estos trabajadores trabajan para grandes empresas (70\%), poseen jornada laboral completa (85\%), contrato indefinido (65\%), son supervisados (90\%) y no supervisan (90\%). Su formación es básica (93\%) y son ligeramente más hombres que mujeres (51\%). El segundo grupo se forma también con empleados de servicio pero lo que es más interesante para nuestro análisis es que está formado por trabajadores manuales semi-cualificados (15\%) o no cualificados (14\%). También, está constituido por administrativos (11\%). Este segmento se compone fundamentalmente de trabajadores industriales con baja cualificación.

El tercer grupo está compuesto sólo por un $10 \%$ de nuestros integrantes. Son trabajadores con un nivel de estudios que supera la educación obligatoria (63\%), tienen jornada completa (95\%), contrato indefinido (95\%) y trabajan también en grandes burocracias públicas (88\%). No son supervisados (38\%) y en gran parte supervisan (67\%). Son hombres en un $70 \%$ y en un $25 \%$ obtienen prestaciones adicionales. Este tercer grupo lo constituyen directivos (7\%), profesionales (24\%) y pequeña burguesía (8\%).

El cuarto grupo lo constituyen el $14 \%$ del total. Son principalmente trabajadores del sector público (95\%). No son supervisados (90\%) y tienen jornada completa (89\%). Están sujetos a un tipo de contrato indefinido (85\%) y un $40 \%$ tiene educación post-obligatoria. Un $58 \%$ son mujeres. Están representados principalmente por profesionales (38\%) y administrativos (18\%).

Por último, el quinto grupo está formado por un 34\% del total, siendo así el grupo mayoritario. Su perfil se compone de aquellos que trabajan en sector privado (100\%), no son supervisados (100\%) y poseen educación post-obligatoria ( $8 \%)$. Son $58 \%$ hombres y el $65 \%$ trabajan en pequeños establecimientos. Este grupo se corresponde principalmente con los pequeños empresarios (40\%). La Tabla 7 resume la información respecto a las lógicas de empleo resultantes.

\section{Tercer paso. Análisis de regresión lineal múltiple}

A continuación, se exponen los resultados del análisis de regresión lineal múltiple. Dada la cantidad de variables contenidas tanto en el índice como en los factores latentes ${ }^{7}$, este análisis sólo introduce como variable de control la edad. El sexo no se ha incorporado ya que está contenido en el análisis de clases latentes, su inclusión crearía problemas graves de endogeneidad. En la Tabla 8 se exponen los resultados del índice por cada lógica laboral. Como se aprecia con respecto a nuestra categoría de referencia correspondiente a la lógica bajo post-industrial, todas las subsiguientes lógicas han resultado ser significativas. En relación a la dirección del coeficiente, los trabajadores ubicados en la lógica bajo industrial presentan una menor calidad intrínseca. En cambio, el resto de las lógicas disfrutan de una mayor calidad. Esto está en sintonía con la primera de nuestras hipótesis, que predecía una mayor calidad de los trabajos relacionados con la lógica post-industrial. No obstante, no se encuentran esta misma división a medida que las lógicas de empleo contemplan profesiones de mayor cualificación. La puntuación con mayor calidad intrínseca se encuentra en la lógica mixta de alta cualificación, formada en su mayor parte por directivos

7 Habría que recordarle a los lectores que para evitar cualquier grado de endogeneidad no existe ninguna variable compartida por el índice y los factores latentes. 
y altos profesionales. En segundo lugar, se encuentra la lógica profesional y, en tercer lugar, la lógica independiente, formada mayoritariamente por pequeños empresarios y autónomos. Hay que señalar que entre estos tres últimos grupos las diferencias no son estadísticamente significativas.

Tabla 7. Características de las lógicas de empleo resultantes

\begin{tabular}{|c|c|c|c|c|c|}
\hline \multirow{2}{*}{ Características } & \multicolumn{5}{|c|}{ Factor latente } \\
\hline & $\begin{array}{l}\text { Bajo Post- } \\
\text { industrial }\end{array}$ & $\begin{array}{c}\text { Bajo } \\
\text { Industrial }\end{array}$ & Mixto & Profesional & Independiente \\
\hline Ocupación & $\begin{array}{c}\text { Servicio } \\
\text { cualificado } \\
\text { Service no } \\
\text { cualificado. }\end{array}$ & $\begin{array}{c}\text { Manual } \\
\text { cualificado } \\
\text { Manual no } \\
\text { cualificado }\end{array}$ & $\begin{array}{l}\text { Directivos y } \\
\text { profesionales }\end{array}$ & Profesionales & $\begin{array}{l}\text { Autónomos, } \\
\text { pequeña b. }\end{array}$ \\
\hline $\begin{array}{l}\text { Tamaño } \\
\text { empresa }\end{array}$ & Pequeño & Grande & Grande & Grande & Pequeño \\
\hline Supervisa & No & No & Sí & No & No \\
\hline Activo & $\begin{array}{l}\text { No post- } \\
\text { obligatoria }\end{array}$ & $\begin{array}{l}\text { No post- } \\
\text { obligatoria }\end{array}$ & $\begin{array}{l}\text { Sí post- } \\
\text { obligatoria }\end{array}$ & $\begin{array}{l}\text { Sí post- } \\
\text { obligatoria }\end{array}$ & $\begin{array}{l}\text { No post- } \\
\text { obligatoria }\end{array}$ \\
\hline Jornada & Parcial & Completa & Completa & Completa & Completa \\
\hline Contrato & Temporal & Indefinido & Indefinido & Indefinido & Indefinido \\
\hline Género & $\begin{array}{l}\text { Mujer } \\
(68 \%)\end{array}$ & $\begin{array}{l}\text { Hombre y } \\
\text { mujer }\end{array}$ & Hombre (70\%) & $\begin{array}{l}\text { Mujer (58\%) y } \\
\text { hombre }\end{array}$ & $\begin{array}{c}\text { Hombre (58\%) y } \\
\text { mujer }\end{array}$ \\
\hline
\end{tabular}

Fuente: elaboración propia con datos de la EWCS (2015)

Para conocer cuál es el efecto de los diferentes grupos que forman las lógicas laborales sobre la calidad intrínseca del trabajo hemos realizado también un análisis de los efectos marginales.

Los resultados del gráfico muestran que todas las lógicas de empleo resultan significativas respecto a la calidad intrínseca del trabajo y que ésta aumenta con el incremento de la edad de los trabajadores, lo que estaría en la linea de investigaciones que muestran una mayor precariedad laboral para el colectivo de trabajadores jóvenes (López Calle, 2019; Muñoz-Comet y Martínez-Pastor, 2017).

Los trabajadores que se ubican en la lógica mixta son los que mayor puntuación han obtenido en el índice de calidad intrínseca, seguidos de los trabajadores ubicados en la lógica profesional. Las lógicas de empleo vinculadas a una mayor cualificación, o con mayor peso de los activos específicos, son las que en nuestro modelo disfrutan de mayores beneficios intrínsecos en el desarrollo de la actividad laboral. Le sigue muy de cerca la lógica independiente, formada en su mayoría por trabajadores autónomos y pequeña burguesía, aunque a edades tempranas se sitúa por debajo de la media, la calidad intrínseca aumenta conforme se alcanza una edad de mayor madurez laboral. Para finalizar, las lógicas bajo post-industrial y bajo industrial se sitúan por debajo de la media de calidad intrínseca del trabajo, siendo esta última la que peor calidad intrínseca presenta de todas las lógicas laborales. 
Tabla 8. Resultados del análisis de regresión múltiple

\begin{tabular}{cc}
\hline Lógicas laborales & ICIT \\
\hline Lógica Baja Post-Industrial & Cat. Referencia \\
Lógica Baja Industrial & $-0.103^{* *}$ \\
Lógica Mixta & $(-2.89)$ \\
Lógica Profesional & $0.182^{* * *}$ \\
& $(4.31)$ \\
Lógica Independiente & $0.155^{* * *}$ \\
& $(3.97)$ \\
Edad & $0.120^{* *}$ \\
cons & $(3.22)$ \\
$\mathrm{N}$ & 0.000104 \\
$* \mathrm{p}<0.05, * *$ & $(0.41)$ \\
\end{tabular}

Fuente: elaboración propia con datos de la EWCS (2015)

Figura 1. Efectos marginales del análisis de regresión lineal

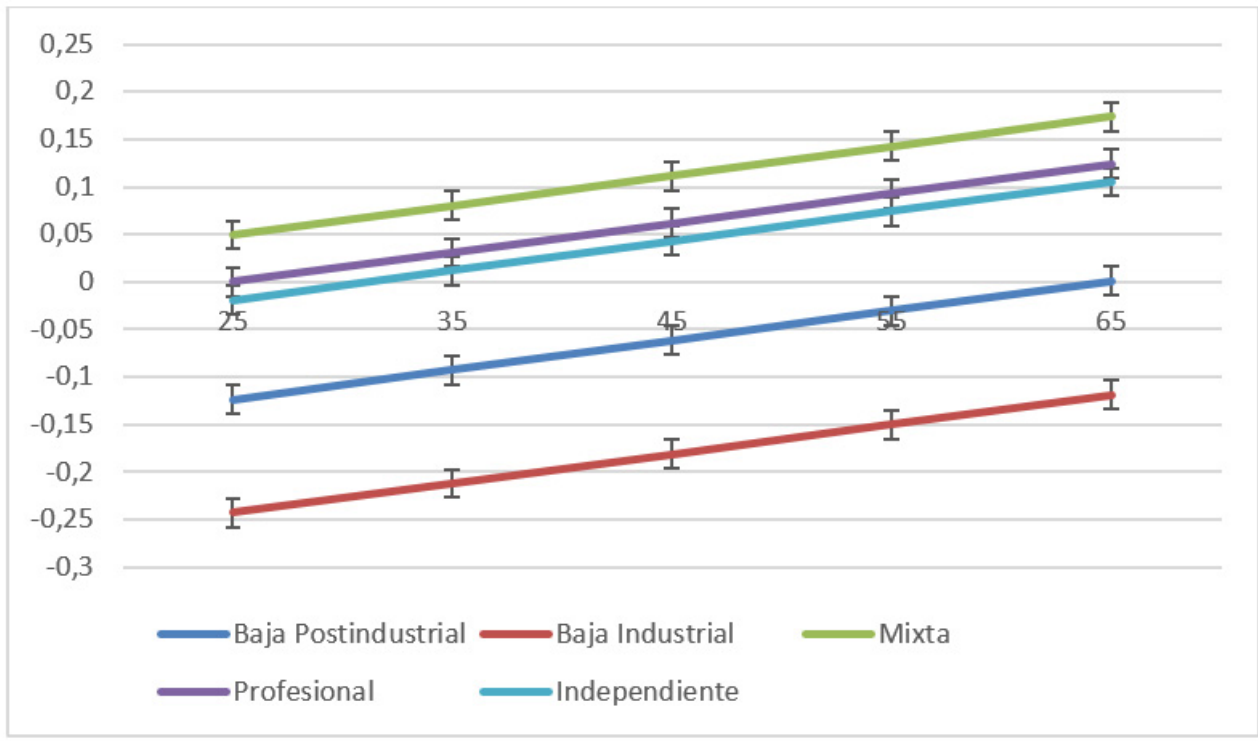

Fuente: elaboración propia con datos de la EWCS (2015) 


\section{CONCLUSIONES}

Con este trabajo hemos vinculado la calidad intrínseca del trabajo con las dos lógicas laborales propias de las sociedades contemporáneas: la industrial y la post-industrial.

Para ello, en primer lugar, hemos construido y validado un Índice de Calidad Intrínseca del Trabajo. Hemos obtenido un total de cuatro dimensiones que pasamos a enumerar: autonomía, interacción, intensidad y sentido del trabajo. La autonomía representa la capacidad del trabajador de tomar de decisiones; la interacción, la calidad de las relaciones entre los trabajadores; la intensidad, el esfuerzo que deben realizar los trabajadores en el desempeño de su actividad; y el sentido, la utilidad social de la actividad realizada.

En segundo lugar, tomando como guía la literatura sobre lógicas de empleo (EspingAndersen, 1993,1999; Hertel, 2017; Oesch, 2006), hemos seleccionado un conjunto de variables que dan cuenta de ellas. Estas variables han sido: tipo de empleo, capacidad de supervisión, cualidad de ser supervisado, tipo de jornada, tipo de contrato, género, tamaño de la empresa, educación y beneficios adicionales. Mediante un análisis de clases latentes han sido extraídas cinco lógicas laborales: post-industrial de baja cualificación, industrial de baja cualificación, mixta, profesional, e independiente. La primera de ellas concentra en su mayoría a mujeres que trabajan con contratos precarios en el sector servicios. La segunda a trabajadores de servicio e industriales con mejores condiciones laborales (contratos indefinidos y jornada completa). En tercer lugar, se encuentra la lógica mixta formada por directivos y profesionales. La hemos denominado mixta porque incluye las categorías más altas de ambas lógicas laborales, tanto la industrial como la post-industrial. Los integrantes de este grupo trabajan en grandes burocracias, tienen jornada completa y tienen algún cargo de autoridad. En cuarto lugar, en la lógica que hemos denominado profesional se ubican los cuadros medios profesionales. En quinto lugar, se halla la lógica independiente que clasifica principalmente a aquellos que tienen un empleo propio, principalmente autónomos, trabajan en empresas pequeñas y no tienen altos activos específicos, en su mayor porcentaje no poseen nivel de enseñanza post-obligatoria.

Por último, hemos vinculado el Índice de Calidad Intrínseca con las cinco lógicas de empleo resultantes del análisis de clases latentes. Los resultados apuntan a una mayor heterogeneidad en la calidad intrínseca para aquellas lógicas cuyas tareas son de naturaleza manual. La lógica mixta, que se caracteriza por aunar la lógica industrial y postindustrial en sus posiciones de mayor privilegio, es a su vez la que puntúa más alto en calidad intrínseca.

Es importante destacar cinco ideas a modo de conclusión para este artículo.

En primer lugar, las lógicas de empleo tienen una naturaleza tanto vertical como horizontal. Encontramos diferencias jerárquicas en lo referente a lo lógica laboral mixta respecto a la bajo industrial no cualificada, así como diferencias en un mismo nivel, como es la lógica bajo industrial respecto a la bajo post-industrial, ambas se caracterizan por contener una menor cualificación en sus ocupaciones.

En segundo lugar, en relación a las lógicas de baja cualificación, la lógica bajo industrial disfruta de una menor calidad intrínseca del trabajo que la lógica bajo post-industrial. En lo que concierne a la autonomía, pese a que los trabajadores de servicio no gozan de una gran capacidad de decisión, las labores del trabajador industrial están sujetas a un mayor control. En relación a las interacciones, la lógica de servicio está vinculada a las relaciones cara a cara, principalmente con los clientes. En lo tocante a la intensidad, los trabajadores industriales están expuestos a una mayor carga de trabajo y esfuerzo físico. Por último, en el sentido del trabajo realizado, las profesiones de servicio tienen de media 
un mayor nivel de estatus que las industriales. Su reputación y prestigio es mejor que la de los trabajadores industriales (Ganzeboom, Graaf, Treiman y Leeuw, 1992).

En tercer lugar, la lógica mixta, a saber, aquella formada por directivos y profesionales de alto rango tiene una mayor calidad intrínseca del trabajo. No obstante, el hecho de que se hallan combinado ambas lógicas en un mismo grupo implica que no existe una lógica industrial diferente a la postindustrial en los niveles superiores, al menos en lo que se refiere a la calidad intrínseca del trabajo.

En cuarto lugar, como en otras tipologías de clase social, hemos descubierto una lógica eminentemente independiente, formada por pequeños empresarios y autónomos. Su puntuación en el índice no es baja. Hay que tener en cuenta que gran parte de la clase creativa (Florida, 2010) está formada por pequeños empresarios.

Por último, habría que resaltar lo profundamente estratificado que están los grupos por sexo. Las mujeres representan casi un $70 \%$ del empleo no cualificado del sector servicio, que presenta puntuaciones por debajo de la media en calidad laboral intrínseca. En cambio en el sector mixto, que goza de mejores condiciones laborales y de una mayor calidad intrínseca del trabajo, los hombres alcanzan el $70 \%$.

\section{REFERENCIAS}

Alonso, L. E. y Fernández Rodríguez, C. J. (2013). Los Discursos del Management: Una Perspectiva Crítica. Lan Harremanak, 28, 42-69.

Alós Moner, R. (2008). El Sindicalismo ante la Acción Colectiva. Cuaderno de Relaciones Laborales, 26(1), 123-148.

Alós Moner, R. y Jódar, P. (2005). Relaciones Laborales Segmentadas en Mercados de Trabajo Segmentados: Algunas Repercusiones en la Calidad de Vida Laboral y en la Participación Sindical. Gaceta Sindical. Reflexión y Debate, (5), 223-249.

Anker, R., Chernyshev, I., Egger, P., Mehran, F. y Ritter, J. A. (2003). La Medición del Trabajo Decente con Indicadores Estadísticos. Revista Internacional del Trabajo, 122(2), 161-195. https://doi.org/10.1111/j.1564-913X.2003.tb00172.x

Bauer, T. K. (2004). High Performance Workplace Practices and Job Satisfaction: Evidence from Europe. IZA RWI-Mitteilungen, 55(1), 57-85.

Bell, D. (1973). El Advenimiento de la Sociedad Post-industrial. Madrid: Alianza

Bericat, E. (2014). The Socioemotional Well-Being Index (SEWBI): Theoretical Framework and Empirical Operationalisation. Social Indicators Research, 119(2), 599-626. https://doi.org/10.1007/s11205-013-0528-z

Bericat, E. (2018). Excluidos de la Felicidad: La Estratificación Social del Bienestar Emocional en España. Madrid: CIS.

Bericat, E. y Cascales Mira, M. (2019). Job Quality. En E. Bericat y M. L. Jiménez-Rodrigo (eds.), The Quality of European Societies: A Compilation of Composite Indicators (pp. 167-186). Switzerland: Springer.

Bescond, D., Châtaignier, A. y Mehran, F. (2003). Seven Indicators to Measure Decent Work: An International Comparison. International Labour Review, 142(2), 179-212. https://doi.org/10.1111/j.1564-913X.2003.tb00258.x 
Bonnet, F., Figueiredo, J. B. y Standing, G. (2003). A Family of Decent Work Indexes. International Labour Review, 142(2), 213-238. https://doi.org/10.1111/j.1564-913X.2003. tb00259.x

Cascales Mira, M. (2010). Análisis de la Satisfacción Laboral en España. Documentos de trabajo (Centro de Estudios Andaluces), 5, 1-41.

Cea D’Ancona, M. A. (2002). Análisis Multivariable Teoría y Práctica de la Investigación Social. Madrid: Síntesis.

Dahl, S. Å., Nesheim, T. y Olsen, K. M. (2009). Quality of Work: Concept and Measurement. SSRN Electronic Journal, 5, 28. https://doi.org/10.2139/ssrn.1489881

Davoine, L., Erhel, C. y Guergoat, M. (2008a). A Taxonomy of European Labour Markets Using Quality Indicators. HAL, University Paris1 Pantheon-Sorbonne (Post-Print and Working Papers), 118.

Davoine, L., Erhel, C. y Guergoat, M. (2008b). Monitoring Quality in Work: European Employment Strategy Indicators and Beyond. International Labour Review, 147(2-3), 163198. https://doi.org/10.1111/j.1564-913X.2008.00030.x

Doeringer, P. B. y Piore, M. J. (1983). Los Mercados Internos de Trabajo. En L. Toharia (ed.), El Mercado de Trabajo: Teorías y Aplicaciones. Lecturas Seleccionadas (pp. 341-368). Madrid: Alianza.

Easterlin, R. A. (1974). Does Economic Growth Improve the Human Lot? Some Empirical Evidence. En Nations and Households in Economic Growth (pp. 89-125). Pensilvania: Elsevier. https://doi.org/10.1016/B978-0-12-205050-3.50008-7

Escobar, M. (2011). La Calidad Democrática. Una Propuesta para su Medición por Expertos. Reis, 133, 59-80. https://doi.org/10.5477/cis/reis.133.59

Escobedo Portillo, M. T., Hernández Gómez, J. A., Estebané Ortega, V. y Martínez Moreno, G. (2016). Modelos de Ecuaciones Estructurales: Características, Fases, Construcción, Aplicación y Resultados. Ciencia \& trabajo, 18(55), 16-22. http://dx.doi.org/10.4067/ S0718-24492016000100004

Esping-Andersen, G. (1992). Post-industrial Class Structures: An Analytical Framework. Estudio/Working Paper 1992/38, 36.

Esping-Andersen, G. (1993). Changing Classes: Stratification and Mobility in Post-industrial Societies. London: Sage Publications.

Esping-Andersen, G. (1999). Social Foundations of Postindustrial Economies. Oxford: Oxford University Press.

Eurofound (2012a). Trends in Job Quality in Europe: A Report Based on the Fifth European Working Conditions Survey. Luxembourg: Publications Office of the European Union.

Eurofound (2012b). Health and Well-being at Work: A Report Based on the Fifth European Working Conditions Survey. Dublin: Eurofound.

Eurofound (2014). Occupational Profiles in Working Conditions: Identification of Groups with Multiple Disadvantages. Dublin: Eurofound.

Eurofound (2015). Sixth European Working Conditions Survey. Publications Office of the European Union, Luxembourg.

Eurofound (2016). Sixth European Working Conditions Survey-Overview Report. Luxembourg: Publications Office of the European Union. 
Fernández-Macías, E. (2012). Job Polarization in Europe? Changes in the Employment Structure and Job Quality, 1995-2007. Work and Occupations, 39(2), 157-182. https://doi.org/10.1177/0730888411427078

Fernández Rodríguez, C. J. (2007). Vigilar y Organizar: Una Introducción a los Critical Management Studies. Madrid: Siglo XXI.

Fernández Rodríguez, C. J. (2016). Estudios Críticos de la Gestión: Estudios Culturales de los Conflictos en el Mundo del Trabajo. Política y Sociedad, 54(1), 23-44. https://doi.org/10.5209/POSO.52063

Fernández Rodríguez, C. J. y Medina-Vicent, M. (2017). Los Nuevos Discursos del Management: Difusión, Impactos y Resistencias. Recerca, 20, 7-14. https://doi.org/10.6035/ Recerca.2017.20.1

Florida, R. (2010). La Clase Creativa. La Transformación de la Cultura del Trabajo y el Ocio en el Siglo XXI. Barcelona: Paidós

Gallie, D. (2007). Welfare Regimes, Employment Systems and Job Preference Orientations. European Sociological Review, 23(3), 279-293. http://doi.org/10.1093/esr/jcm001

Gallie, D., Felstead, A. y Green, F. (2012). Job Preferences and the Intrinsic Quality of Work: The Changing Attitudes of British Employees 1992-2006. Work, Employment and Society, 26(5), 806-821. https://doi.org/10.1177/0950017012451633

Gallie, D., Felstead, A., Green, F. e Inanc, H. (2014). The Quality of Work in Britain over the Economic Crisis. International Review of Sociology, 24(2), 207-224. https://doi.org/10.108 0/03906701.2014.933023

Gallie, D., Zhou, Y., Felstead, A., Green, F. y Henseke, G. (2017). The Implications of Direct Participation for Organisational Commitment, Job Satisfaction and Affective Psychological Well-Being: A Longitudinal Analysis: Implications of Direct Participation: Longitudinal Analysis. Industrial Relations Journal, 48(2), 174-191. https://doi.org/10.1111/irj.12174

Ganzeboom, H. B. G., Graaf, P. M. de, Treiman, D. J., Leeuw, J. de (1992). A Standard International Socioeconomic Index of Occupational Status. Tilburg: Work and Organization Research Centre.

Ghai, D. P. (2002). Decent work: Concepts, Models and Indicators. Geneva: International Institute for Labour Studies.

Green, F. (2006). Demanding work: The Paradox of Job Quality in the Affluent Economy. Princeton: Princeton University Press.

Green, F. y Mostafa, T. (2012). Job Quality Indices for Europe. A Report Based on The Fifth European Working Conditions Survey. London: Llakes Centre/Institute of Education.

Hertel, F. R. (2017). Social Mobility in the 20th Century: Class Mobility and Occupational Change in the United States and Germany. Wiesbaden: Springer.

Hochschild, A. R. (1979). Emotion Work, Feeling Rules, and Social Structure. The American Journal of Sociology, 85(3), 551-575.

Hurley, J., Storrie, D. W. y Jungblut, J. M. (2011). Shifts in the Job Structure in Europe During the Great Recession. Luxembourg: Publications Office of the European Union.

Jisu, H., Delorme, D. E. y Reid, L. N. (2006). Perceived Third-Person Effects and Consumer Attitudes on Prevetting and Banning DTC Advertising. Journal of Consumer Affairs, 40(1), 90-116. https://doi.org/10.1111/j.1745-6606.2006.00047.x 
Kalleberg, A. L. (1977). Work Values and Job Rewards: A Theory of Job Satisfaction. American Sociological Review, 42(1), 124. https://doi.org/10.2307/2117735

Kalleberg, A. L. (2000). Nonstandard Employment Relations: Part-Time, Temporary and Contract Work. Annual Review of Sociology, 26, 341-365. https://doi.org/10.1146/annurev. soc.26.1.341

Kalleberg, A. L. (2011). Good Jobs, Bad Jobs: The Rise of Polarized and Precarious Employment Systems in the United States, 1970s to 2000s. New York: Russell Sage Foundation.

Kalleberg, A. L. y Vaisey, S. (2005). Pathways to a Good Job: Perceived Work Quality among the Machinists in North America. British Journal of Industrial Relations, 43(3), 431-454. https://doi.org/10.1111/j.1467-8543.2005.00363.x

Kaufman, L. y Rousseeuw, P. J. (1990) Partitioning around Medoids (Program PAM). En Finding Groups in Data: An Introduction to Cluster Analysis (pp. 68-125). Hoboken: John Wiley \& Sons.

Lahera Sánchez, A. (2004). La Participación de los Trabajadores en la Calidad Total: Nuevos Dispositivos Disciplinarios de Organización del Trabajo. Reis, 106(04), 63-101. https://doi.org/10.2307/40184585

Leschke, J. y Watt, A. (2008). Putting a Number on Job Quality? Constructing a European Job Quality Index. Luxembourg: ETUI.

López Calle, P. (2019). Subjetividad Precaria como Recurso Productivo. Crisis, Trabajo e Identidad en las Periferias Metropolitanas Desindustrializadas. Revista Española de Sociología, 28(2), 347-364. http://dx.doi.org/10.22325/fes/res.2018.56

Magidson, J. y Vermunt, J. K. (2004). Latent Class Models. En D. Kaplan (ed.), The Sage Handbook of Quantitative Methodology for the Social Sciences (pp. 175-198). Thousand Oaks: Sage Publications.

Martel, J. P. y Dupuis, G. (2006). Quality of Work Life: Theoretical and Methodological Problems, and Presentation of a New Model and Measuring Instrument. Social Indicators Research, 77(2), 333-368. https://doi.org/10.1007/s11205-004-5368-4

Monroy, L., Vidal, R. y Saade, A. (2009). Análisis de Clases Latentes: una Técnica para Detectar Heterogeneidad en Poblaciones. Cuaderno técnico 2. México: Centro Nacional de Evaluación para la Educación Superior

Muñoz-Comet, J. y Martínez-Pastor, J. I. (2017). ¿Es la Precariedad Igual para Todos los Trabajadores Temporales? Diferencias y Semejanzas entre Clases Sociales. Revista Española de Sociología, 26(2), 169-184. https://doi.org/10.22325/fes/res.2017.11

Muñoz de Bustillo Llorente, R., Fernández Macías, E., Antón, J. I. y Esteve, F. (2009). Indicators of Job Quality in the European Union. Bruselas: European Parliament.

Muñoz de Bustillo Llorente, R., Fernández Macías, E., Antón, J. I. y Esteve, F. (eds.). (2011a). Measuring More than Money: The Social Economics of Job Quality. Cheltenham: Edward Elgar.

Muñoz de Bustillo Llorente, R., Fernández Macías, E., Esteve Mora, F. y Antón Pérez, J. I. (2011b). E Pluribus Unum? A Critical Survey of Job Quality Indicators. Socio-Economic Review, 9(3), 447-475. https://doi.org/10.1093/ser/mwr005

Oesch, D. (2006). Redrawing the Class Map: Stratification and Institutions in Britain, Germany, Sweden, and Switzerland. New York: Palgrave Macmillan. 
Pérez Zapata, O., Álvarez Hernández, G. y Castaño Collado, C. (2017). Engagement y/o Intensificación del Trabajo ¿Opción y/o Obligación?: «Si no haces lo que te gusta, te tiene que gustar lo que haces». Política y Sociedad, 54(3), 707-732. https://doi.org/10.5209/ POSO.52176

Pérez-Zapata, O., Pascual, A. S., Alvarez-Hernández, G. y Collado, C. C. (2016). Knowledge Work Intensification and Self-management:The Autonomy Paradox. Work Organisation, Labour and Globalisation, 10(2), 27-49. https://doi.org/10.13169/workorgalaboglob.10.2.0027

Piore, M. J. (1983). Notas para una Teoría de la Estratificación del Mercado de Trabajo. En L. Toharia (ed.), El Mercado de Trabajo: Teorías y Aplicaciones; Lecturas Seleccionadas (pp. 193-221). Madrid: Alianza.

Piore, M. J. y Sabel, C. F. (1990). La Segunda Ruptura Industrial. Madrid: Alianza.

Polavieja, J. G. (2003). Estables y Precarios. Desregulación Laboral y Estratificación Social en España. Madrid: CIS.

Requena, M. y Stanek, M. (2015). Las Clases Sociales en España: Cambio, Composición y Consecuencias. En Informe España 2015: Una interpretación de su realidad social (pp. 487-517). Madrid: Fundación Encuentro.

Royuela, V., López-Tamayo, J. y Suriñach, J. (2008). The Institutional vs. The Academic Definition of the Quality of Work Life. What is the Focus of the European Commission? Social Indicators Research, 86(3), 401-415. https://doi.org/10.1007/s11205-007-9175-6

Santos Ortega, J. A. (2012). «La Bolsa y la Vida»: Efectos de la Lógica Financiera sobre los Mercados de Trabajo Precarios. En L. E. Alonso Benito y C. J. Fernández Rodríguez (eds.), La Financiarización de las Relaciones Salariales (pp. 127-157). Madrid: Los libros de la catarata.

Seeman, M. (1967). On the Personal Consequences of Alienation in Work. American Sociological Review, 32(2), 273-285. https://doi.org/10.2307/2091817

Tangian, A. (2007). Analysis of the Third European Survey on Working Conditions with Composite Indicators. European Journal of Operational Research, 181(1), 468-499. https://doi.org/10.1016/j.ejor.2006.05.038

\section{NOTA BIOGRÁFICA}

María Cascales es Doctora en Sociología por la Universidad de Sevilla y desde el 2010 ha trabajado como profesora interina en el Departamento de Sociología de la misma. Pertenece al grupo de investigación de Estudios Sobre la Calidad de las Sociedades Europeas (ECALSE), resultando la publicación del libro The Quality of European Societies: A Compilation of Composite Indicators (Springer, 2019). Ha participado en la red INCASI( International Network for Comparative Analysis of Social Inequalities) financiada por la Comisión Europea, realizando varias estancias de investigación en Latinoamérica, y en la actualidad participa en el proyecto de investigación "Dinámicas de movilidad social en España" (DINAMOS) vinculado al proyecto INCASI. 
Ildefonso Marqués-Perales es profesor contratado doctor del Departamento de Sociología de la Universidad de Sevilla. Es doctor en Sociología por la Universidad Pontificia de Salamanca (2005). Tesis: Génesis de la teoría social de Pierre Bourdieu. Ha sido investigador del Centro de Estudios Andaluces (2008-2014) y profesor titular de la Escuela de Trabajo Social, Turismo y Relaciones Laborales (2000-2008). Entre 2016 y 2019 ha sido investigador principal por la Universidad de Sevilla del Proyecto INCASI «International Network for Comparative Analysis of Social Inequalities» RISE-Horizon 2020. Su principal campo de estudio son las clases sociales en España. Es autor del libro La movilidad social en España (2015) y de varios artículos, entre los que se encuentran: «Intergenerational Social Mobility in Spain between 1956 and 2011: The role of educational expansion and economic modernization in a late industrialized country» (con Gil-Hernández y Fachelli, 2017); "Social mobility and demand for redistribution in Europe: a comparative analysis» (con Jaime Castillo, 2019); «Relevancia de la heterogeneidad socioeconómica. Estudio comparativo entre América Latina y Europa basado en la adaptación del esquema EGP» (con Chávez-Molina, 2019). 